
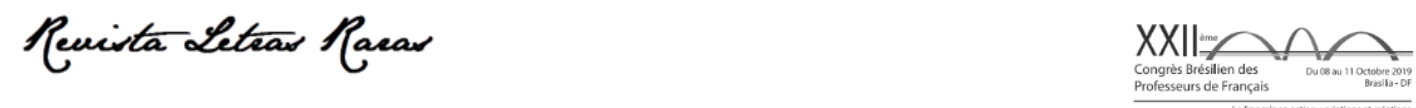

Actes du XXIlème Congrès brésilien des professeurs de français (dans Édition spéciale de la Revue Letras Raras 2020)

ISSN : 2317-2347 - v. 9, Dossier Spécial (2020)

Todo o conteúdo da RLR está licenciado sob Creative Commons Atribuição 4.0 Internacional

\title{
Les approches intercompréhensives : une pédagogie au service du plurilinguisme
}

\section{Claudine Franchon ${ }^{*}$}

Doctorat en Sciences du langage à l'Université Stendhal - Grenoble 3. Professeure Associée I à l'Université de Brasilia (UnB).

iD https://orcid.org/0000-0002-0442-6581

Reçu le 11 oct. 2020. Approuvé le 14 nov. 2020.

\section{Comment citer cet article:}

FRANCHON, Claudine. Les approches intercompréhensives: une pédagogie au service du plurilinguisme. In: CONGRES BRESILIEN DES PROFESSEURS DE FRANÇAIS, 22., 2019, Brasília. Actes du XXIIème Congrès Brésilien des Professeurs de Français. Édition spéciale de la Revue Letras Raras: Campina Grande. EDUFCG. nov. 2020, p. 243-255.

\section{RÉSUMÉ}

Cet article propose une réflexion sur l'usage des approches intercompréhensives (AIC) dans l'enseignement/apprentissage, sur la base d'une éducation aux langues novatrice qui mobilise les expériences linguistiques et communicatives de l'apprenant dans le cadre d'un enseignement/apprentissage basé sur la construction de nouvelles connaissances en langues génétiquement apparentées. Notre communication sous la forme d'un compte-rendu des interventions qui ont eu lieu dans le cadre du symposium dédié à l'intercompréhension (IC) permettra de mettre en lumière différents itinéraires pédagogiques de l'alternance des langues romanes dans la perspective d'un décloisonnement pédagogique dans la formation en langues, sur des terrains diversifiés, dans une dynamique interdisciplinaire, dans différents contextes éducatifs. Les contributions, au nombre de huit, ont abordé l'intercompréhension dans ses dimensions éducatives tant au niveau scolaire (primaire et secondaire) qu'universitaire ou plus spécifiquement dans le cadre de la formation professionnelle pour adultes

MOTS-CLÉS : Intercompréhension; Langues romanes; Enseignement-apprentissage ; Éducation linguistique; Environnement virtuel et remédiation

\section{L'intercompréhension : Les éléments clefs d'un concept extensible et malléable}

Suscitant de nombreuses recherches en didactique depuis les années 1990, l'intercompréhension (IC) et plus largement les « approches intercompréhensives » (AIC) sont devenues au fil des décennies une véritable voie didactique en matière d'apprentissage/enseignement des langues, ne se bornant plus à des initiatives ponctuelles de l'ordre de la sensibilisation. En effet, comme le constatent Degache et Garbarino « le nombre d'actions pédagogiques qui mettent en œuvre une approche intercompréhensive de façon durable,

\laudine unb@yahoo.br 

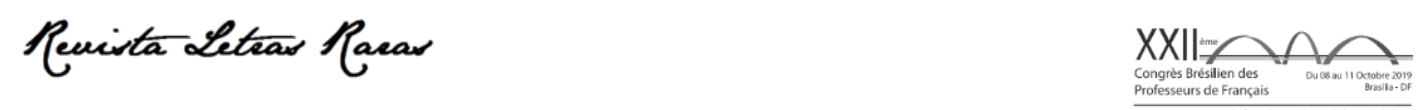

Actes du XXIlème Congrès brésilien des professeurs de français (dans Édition spéciale de la Revue Letras Raras 2020) ISSN : 2317-2347 - v. 9, Dossier Spécial (2020)

Todo o conteúdo da RLR está licenciado sob Creative Commons Atribuição 4.0 Internacional

au moins comme une partie de cours ou d'une formation, est en hausse constante ». (DEGACHE ; GARBARINO, 2017, p. 8).

Dans le cadre de la didactique des langues voisines, l'intercompréhension (IC) relève de ce que Candelier (2007) nomme les approches plurielles et que d'autres appellent didactique du plurilinguisme (MOORE ; CAJO, 2009). La spécificité de cette démarche est de travailler sur le contact des langues qui pourra être analysé sous différents angles, dans différents contextes éducatifs, dans différentes structures et environnements, et ce, en fonction des approches méthodologiques mises en œuvre. En ce sens, les recherches et réflexions qui traitent du contact de langues en tant qu'objet d'étude pourront considérer le contact de langues sous plusieurs angles : à partir du contact, vers le contact, autour du contact, avec le contact.

L'intercompréhension (IC), qui est à la fois un objectif à atteindre (se comprendre mutuellement), un ensemble de stratégies de compréhension qui s'appuient sur les ressemblances inter-langues fait l'objet d'un grand nombre de définitions. Parmi plusieurs définitions du concept, nous en retiendrons un certain nombre qui attribue un sens plus complet et moins restrictif à la notion d'intercompréhension (IC) en tablant sur le fait que cette modalité de communication bilingue, trilingue ou d'avantage, peut se concevoir comme un « desenvolvimento de capacidade de co-construção de sentido no encontro entre línguas diferentes e de fazer uso pragmático dessa capacidade num situação concreta ». (CAPUCHO, 2004, p.86).

L'auteur souligne qu'une telle conception favorise l'existence et la co-existence de chacun, permettant l'expression plurilingue et la promotion du respect de la langue d'autrui. Dans ce même ordre d'idée, d'une définition large de l'intercompréhension en didactique des langues en lien avec le colloque IC 2012, Degache et Garbarino retiennent que l'intercompréhension (IC) est un espace interlocutoire où « chacun parle sa propre langue et comprend celle de l'autre, que cela soit spontané ou choisi par les interlocuteurs ». (DEGACHE ; GARBARINO, 2017, p.9).

Par ailleurs, le regard sociologique que pose Blanchet sur l'intercompréhension entre langues différentes le fait revenir sur la définition de ce concept pour en souligner les fondements théoriques et les orientations didactiques extrêmement innovants. Autour de thèmes centraux qu'il définit comme des notions cruciales (langue(s), pluralité linguistique, construction des significations, représentations, acceptabilité, parenté/proximité, éducation interculturelle), il prône une redéfinition du concept de langue et souligne que «L'intercompréhension entre langues différentes, phénomène attesté de façon difficilement contestable, fournit un argument massif 

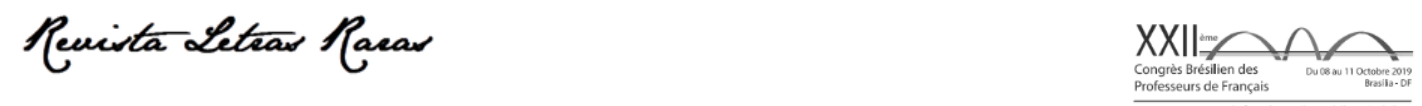

Actes du XXIlème Congrès brésilien des professeurs de français (dans Édition spéciale de la Revue Letras Raras 2020) ISSN : 2317-2347 - v. 9, Dossier Spécial (2020)

Todo o conteúdo da RLR está licenciado sob Creative Commons Atribuição 4.0 Internacional

contre cette façon de caractériser les pratiques linguistiques en langues distinctes. Elle interroge radicalement la notion de frontière entre les langues. [...] Je ne veux pas dire par là que toute catégorisation en langues distinctes, ou, à l'inverse, tout effacement de frontière entre langue soit bon ou mauvais. Je veux dire que ça relativise considérablement la notion de langue comme entité distincte d'une autre et que ça attire notre attention sur les critères effectifs de cette catégorisation qui sont, dans la plupart des cas, d'ordre sociolinguistique et non linguistique (au sens structurolinguistique) ». (BLANCHET, 2018, p. 29-30).

Par ailleurs, les quatre principes fondateurs qui président au développement des compétences langagières dans le cadre de l'intercompréhension (IC) et qui fondent les recherches des auteurs du symposium sont (DEGACHE; GARBARINO, 2017, p.10) :

- la priorité accordée à la réception, aux activités de compréhension, en les dissociant des activités de production langagière, ce à quoi on se réfère généralement comme dissociation des aptitudes (ou des "compétences", ARAUJO E SA; DEGACHE; SPITA, 2010);

- l'importance donnée au répertoire langagier, soit au « déjà là ॥ ;

- le rôle capital accordé aux stratégies de l'apprenant, à l'identification et à la reconnaissance de sa capacité à comprendre des messages dans des langues peu ou pas connues grâce à ses propres démarches, mais aussi à leur optimisation et leur enrichissement ;

- la prise en compte de la parenté linguistique, à la fois comme stratégie de compréhension, d'apprentissage et d'enseignement.

La prise en compte de la proximité génétique des langues, autre principe central, (filiation historique commune et donc des transformations parallèles comparées) est un des aspects les plus fructueux de cette approche, en remettant au premier plan la LV1, dans l'apprentissage d'une ou plusieurs langues proches. Les idiomes romans possèdent, de ce point de vue, une caractéristique qui leur confère un intérêt particulier. Ils bénéficient, en effet, d'une part, de renseignements fort précis sur leur langue commune, le latin, et, d'autre part, l'évolution de plusieurs d'entre eux est bien documentée grâce à des sources écrites.

Cette observation fait, de cette famille de langues génétiquement apparentées, un laboratoire d'explorations particulièrement fécond qui permet de développer un champ d'expertise puissant en didactique des langues. La prise en compte de la parenté linguistique est comme nous 

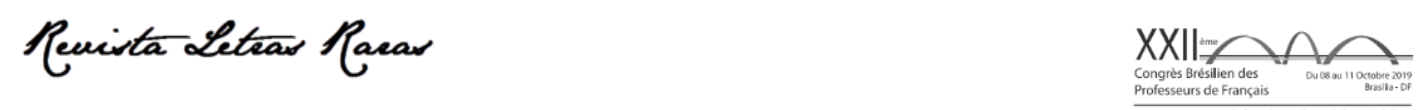

Actes du XXIlème Congrès brésilien des professeurs de français (dans Édition spéciale de la Revue Letras Raras 2020) ISSN : 2317-2347 - v. 9, Dossier Spécial (2020)

Todo o conteúdo da RLR está licenciado sob Creative Commons Atribuição 4.0 Internacional

venons de le souligner une piste fructueuse pour les approches intercompréhensives (AIC). À ce propos, Lewis, Simons et Fennig (2015) observent que lorsque la parenté linguistique entre deux parlers est estimée à au moins $85 \%$ (en particulier au niveau du lexique), on peut alors parler d'inherent intelligibility alors que lorsque la compréhension est rendue possible par l'exposition préalable à la langue ou par l'apprentissage, on parlera alors d'acquired intelligibility, et ce, incompris au-dessous de $85 \%$ de similarité et lorsque les langues ne sont pas apparentées.

La didactique des langues a pris la mesure du potentiel que constitue l'exploitation des situations de contact de langues en termes d'efficacité pédagogique. Ainsi, la facilité des contacts multilingues générés par les apports du numérique et la perspective actionnelle sous l'essor de la diffusion du Cadre européen commun de référence pour les langues (CECR) qui fait loi depuis les années 2000 avec un ancrage résolument social et communicatif travaillent dans ce sens.

II convient de signaler qu'au fil des années nous avons assisté à une évolution du concept d'intercompréhension (IC) en relation avec la visée communicative des processus à développer et les supports didactiques disponibles. Aussi, si l'intercompréhension (IC) était au départ, comme nous l'avons mentionné, liée à des situations de réception écrite, en l'occurrence celle de textes écrits dans différentes langues apparentées, de façon simultanée ou quasi simultanée, cette dimension réceptive du concept (centrée sur un travail cognitif et méta cognitif comparatiste incitant le lecteur à comparer les langues et à les comprendre) a évolué.

L'intercompréhension (IC) s'est intéressée à la dimension orale du langage puis le développement des technologies de l'information et de la communication (TIC) a permis de situer la notion dans le cadre des situations d'interactions plurilingues, (forums et chats). Des programmes de recherche ont ainsi exploité les ressources informatiques pour favoriser et exploiter pédagogiquement les échanges plurilingues à distance. Aussi, la distinction entre intercompréhension entendue au sens réceptif ou au sens interactif est une distinction qui structure à présent le champ conceptuel de l'intercompréhension (IC).

Comme le définit Degache (DEGACHE, 2009, p.84) les interactions plurilingues impliquent que chacun s'exprime dans sa (ses) propre langue (s) de référence et fasse l'effort de comprendre l'autre dans sa (ses) langue(s), ce qui génère des échanges de tout type que Degache et Garbarino résument ainsi : « natif/non natif dans une langue, chacun dans sa première langue, chacun dans la première langue de l'autre, échanges dans une langue tierce, échange en lingua franca ou dans un système linguistique composite de circonstance (un sabir ad hoc)... Comment qualifier ces 

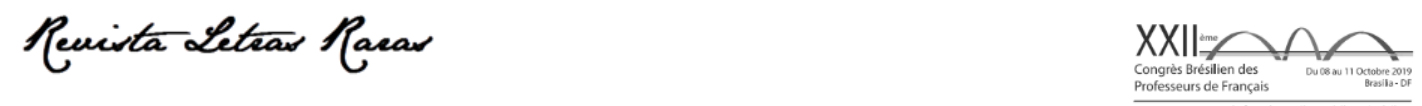

Actes du XXIlème Congrès brésilien des professeurs de français (dans Édition spéciale de la Revue Letras Raras 2020)

ISSN : 2317-2347 - v. 9, Dossier Spécial (2020)

Todo o conteúdo da RLR está licenciado sob Creative Commons Atribuição 4.0 Internacional

différents types d'échanges? Selon quelle typologie des interactions de référence ? Relèvent-ils de la communication exolingue, de la communication endolingue ou d'un autre type d'interaction ? ». (DEGACHE; GARBARINO, 2017, p. 13).

Si l'on retient les critères définis par la littérature linguistique francophone travaillant sur l'appropriation des langues, la nature des échanges concernés par l'intercompréhension (IC) renvoie à la notion de communication ou conversation exolingue qui selon Porquier (1984) est « celle qui s'établit par le langage par des moyens autres qu'une langue maternelle éventuellement commune aux participants» (PORQUIER, 1984, p. 16) par opposition à la communication endolingue entre individus de même langue maternelle. II est à noter que cette définition ouvre le champ à de nombreuses configurations qui vont au-delà de la simple dichotomie natif/non natif dans une langue donnée. Ainsi Py (PY, 1990, p.82) dans une étude de situations exolingues détermine qu'un échange peut être qualifié d'exolingue lorsque l'on observe :

- une « asymétrie importante dans la compétence linguistique des interlocuteurs (la langue utilisée est en principe langue première pour l'un et langue seconde pour l'autre) » ; - une « thématisation récurrente de cette asymétrie - surtout apparente lors de la résolution de difficultés (malentendus, incompréhensions, etc » ;

- une « asymétrie linguistique [qui] est prolongée par des asymétries dans les règles de l'interaction et les conventions culturelles (par exemple dans la manière d'identifier et d'interpréter les indices de contextualisation) ».

Comme le font remarquer Garbarino et Degache (2017), on constate que dans les échanges intergroupes en ligne, et en particulier dans les échanges plurilingues en intercompréhension pour ce qui nous concerne, la complexité et la diversité de la communication augmentent notablement. Matthey et De Pietro (1997) le synthétisent en identifiant quatre types d'interactions verbales :

- $\quad$ endolingue-unilingue (asymétrie linguistique minimale dans une seule langue)

- $\quad$ exolingue-unilingue (natif/non-natif)

- $\quad$ endolingue-bilingue (asymétrie minimale entre les locuteurs dans deux langues ou parler bilingue)

- $\quad$ exolingue-bilingue (deux langues inégalement maîtrisées sont utilisées par les interlocuteurs) 

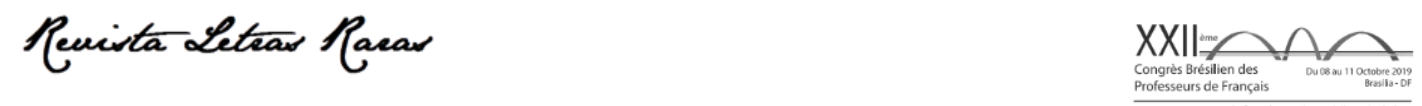

Actes du XXIlème Congrès brésilien des professeurs de français (dans Édition spéciale de la Revue Letras Raras 2020) ISSN : 2317-2347 - v. 9, Dossier Spécial (2020)

Todo o conteúdo da RLR está licenciado sob Creative Commons Atribuição 4.0 Internacional

Garbarino et Degache (2017) soulignent que les interactions recueillies en situation d'intercompréhension, quand chacun s'exprime dans la langue qu'il maîtrise le mieux « constituent une extension de ce dernier type que nous pouvons qualifier d'exolingue-plurilingue puisque plusieurs langues, inégalement maîtrisées, sont utilisées délibérément par les interlocuteurs. II s'agit donc ici, en général, d'interactions entre natifs de différentes langues.

Toutefois, quand ces interactions réunissent des interlocuteurs qui ont des compétences symétriques dans trois langues au moins, ce qui s'avère fréquent dans les interactions écrites en ligne en langues romanes, on peut avoir affaire à des échanges endolingues-plurilingues, par exemple entre Portugais comprenant le français et l'espagnol et Français comprenant l'espagnol et le portugais, le tout avec des compétences symétriques et sans qu'apparaisse bien sûr, dans l'interaction, de marque exolingue. Cela étant, il faudrait encore distinguer un cas, celui de l'échange exolingue-bilingue ou plurilingue où chacun s'exprime dans une autre langue que sa L1». (DEGACHE; GARBARINO, 2017, p.15-16).

Sur la base d'une éducation aux langues «transformante », nous verrons ci-après comment les huit projets présentés dans le cadre du symposium Les approches intercompréhensives : une pédagogie au service du plurilinguisme mettent l'accent sur le développement de stratégies linguistiques transposables qui permettent d'aborder simultanément plusieurs langues romanes montrant ainsi comment l'intercompréhension (IC) et plus largement les approches intercompréhensives (AIC) revêtent toute leur pertinence en matière d'enseignement/apprentissage.

Les auteurs des communications ancrent leur étude dans des contextes institutionnels variés qui concernent des publics différents, où l'appropriation des langues se joue, dans ses dimensions éducatives, tant au niveau scolaire (primaire et secondaire) qu'universitaire ou plus spécifiquement dans le cadre de la formation professionnelle pour adultes.

\section{Présentation des contributions}

Nous avons opté pour une présentation des différents travaux par contexte d'enseignement (scolaire, supérieur, formation d'adultes et professionnelle) en séparant, pour ce qui concerne le supérieur (au niveau de l'université), les actions relevant de l'intercompréhension réceptive et celles relevant de l'intercompréhension interactive. Le contexte universitaire reste ici 

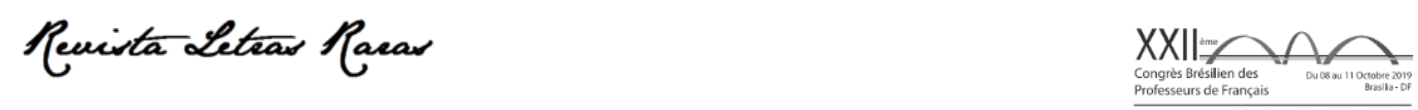

Actes du XXIlème Congrès brésilien des professeurs de français (dans Édition spéciale de la Revue Letras Raras 2020) ISSN : 2317-2347 - v. 9, Dossier Spécial (2020)

Todo o conteúdo da RLR está licenciado sob Creative Commons Atribuição 4.0 Internacional

prépondérant puisque cinq contributions sur huit le concernent mais l'importance des deux autres secteurs manifeste la diversification des terrains de diffusion de l'intercompréhension (IC) dans le domaine des langues romanes.

\subsection{L'intercompréhension réceptive en contexte universitaire}

Les travaux de Livia Miranda de Paulo (présentation du 9 octobre) sous le titre L'intercompréhension dans le cadre de la licence de lettres : la formation des sujets plurilingues à partir de la lecture de textes académiques en langues romanes, ont fait état d'une recherche doctorale, conclue en 2018, focalisée sur l'étude d'un programme de formation de l'Université de São Paulo (USP), qui s'est tenu en 2016 et 2017. Ce programme, basé sur le développement des compétences en lecture de textes à visée académique en langues étrangères s'est adressé à un public de futurs enseignants notamment dont le but était de se former à l'intercompréhension (IC) pour intégrer cette approche dans ses pratiques de cours ainsi qu'à d'autres participants se préparant à la mobilité. Comme l'a souligné l'auteure lors de sa présentation, la question de former un apprenant autonome, conscient et plurilingue, pouvant circuler entre les langues pour atteindre ses objectifs d'apprentissage, a été au centre de la formation dispensée. II s'est agi de mettre en place un travail autour du transfert pour viser la compréhension de textes en langues voisines au moyen de la mobilisation de stratégies connues en langue 1 et en langues 2 et 3 . La chercheuse a souligné comment les activités intercompréhensives de lecture conçues comme moyen de sensibilisation aux langues romanes cibles (espagnol/français/italien) avaient fait surgir chez les apprenants lusophones de nouvelles attitudes et représentations liées au processus de construction du sujet plurilingue (choix d'une méthodologie de type qualitatif sur la base d'une approche émique centrée sur le recueil de significations culturelles liées au point de vue des acteurs pour rendre compte des résultats). Un focus porté sur le choix des textes (genres textuels et identification de la structure textuelle) avec en perspective comment l'étude de genres textuels favorisaient le développement de compétences de lecture en contexte universitaire a été le fil conducteur de cette communication. Les données fournies ont démontré l'amplitude des potentialités de l'intercompréhension (IC).

Thomas De Fornel, quant à lui, nous a présenté une communication (présentation du 9 octobre) intitulée De l'intercompréhension intégrée des langues et cultures romanes pour une 

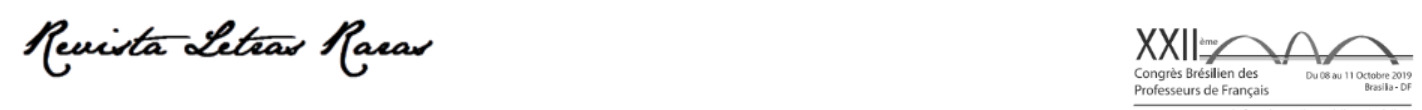

Actes du XXIlème Congrès brésilien des professeurs de français (dans Édition spéciale de la Revue Letras Raras 2020) ISSN : 2317-2347 - v. 9, Dossier Spécial (2020)

Todo o conteúdo da RLR está licenciado sob Creative Commons Atribuição 4.0 Internacional

littératie plurilingue : une étude comparée franco-brésilienne, qui pose les fondamentaux d'une recherche qui sera conduite auprès d'étudiants de Licence et Master de Lettres en France et au Brésil, impliquant les universités du Párana (UFPR) et de Bordeaux. Cette recherche envisage l'intercompréhension comme un outil méthodologique et heuristique dans le cadre de la formation des enseignants. L'auteur construit sa recherche en considérant la notion de littératie qu'il envisage sous l'angle du plurilinguisme, en accord avec les paramètres du cadre de référence pour les approches plurielles (CARAP). Signalons que la notion de littératie, largement circulante, et qui génère le débat entre les tenants d'une perspective "autonome » de la notion et ceux qui la considèrent comme dépendante de son ancrage social, est ici envisagée dans son inscription sociale. Les questions posées par l'auteur, qui prend en compte deux terrains de recherche, un terrain francophone et un terrain lusophone, sont fondamentalement centrées sur une redéfinition de la notion de littératie dans un contexte plurilingue et pluriculturelle d'enseignement des langues. L'auteur envisage ici l'intercompréhension comme une méthodologie robuste qui permettrait de négocier un enseignement/apprentissage de la littératie en langues romanes. La démarche se veut décloisonnant et transversale, nourrie de la diversité des contextes et des champs disciplinaires (études littéraires et étude linguistique). L'auteur a pour ambition de définir une thématique transdisciplinaire adossée à l'intercompréhension des langues romanes.

\subsection{L'intercompréhension interactive en contexte universitaire}

La communication de Yádini Do Canto Winter Dos Santos, D’Andrea Klinger Ramires, de Fatima Ninahuanca et de Sandra Velasquez (présentation du 10 octobre) intitulée Língua Mix : la création de vidéos You Tube pour discuter l'intercompréhension en langues romanes a porté sur la création d'une chaîne YouTube « Lingua Mix » présentant des vidéos d'animation associées à des dialogues enregistrés entre locuteurs de langues romanes (locuteurs francophones, lusophones, hispanophones et italophones). Cette initiative relevant d'un travail élaboré dans le cadre d'une discipline du Master en Didactique des langues de l'Université de Strasbourg a été réalisée par des étudiants hispanophones originaires de Bolivie, du Pérou et de Colombie. Les auteures ont mis l'accent sur leur volonté de mettre en lumière la productivité des parentés linguistiques en tablant sur les vertus d'une approche contrastive particulièrement fructueuse dans le cadre de la famille des langues romanes. Elles ont exposé les fondements didactiques qui 

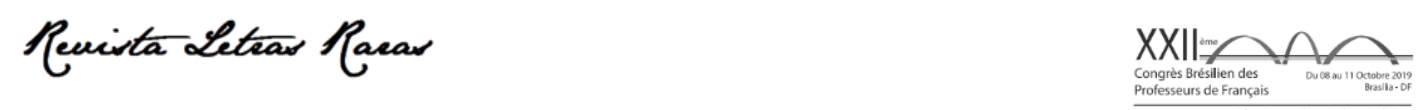

Actes du XXIlème Congrès brésilien des professeurs de français (dans Édition spéciale de la Revue Letras Raras 2020) ISSN : 2317-2347 - v. 9, Dossier Spécial (2020)

Todo o conteúdo da RLR está licenciado sob Creative Commons Atribuição 4.0 Internacional

présidaient à la création de leur produit en insistant sur la progression pédagogique qui soustendait les contenus de cette chaîne en libre accès pour en souligner les bénéfices et l'originalité en termes d'enseignement/apprentissage plurilingue. La présentation des huit vidéos composant le programme a permis de saisir l'articulation et la globalité de la démarche fondée selon les étapes suivantes : 1. vidéo consacrée à la genèse des quatre langues romanes (première vidéo) ; 2 . deux vidéos ayant trait à la problématique de la transparente versus opacité entre les langues génétiquement apparentées (les deux vidéos suivantes) + conversations disponibles entre quatre locuteurs de langues romanes; 3 . cinq vidéos dédiées à des discussions entre des locuteurs de différentes langues romanes où chacun s'exprime exclusivement dans sa langue maternelle (les cinq dernières vidéos).

L'intervention de René Strehler (présentation du 10 octobre) intitulée Revise o seu francês : um applicativo para estudantes de francês como língua estrangeira a porté sur un logiciel élaboré par l'auteur. Cette ressource (perspective bilingue) mise à disposition des étudiants du cours de Lettres-Français de l'Université de Brasilia a une visée de soutien. Lors de sa présentation, l'auteur a relaté les paramètres linguistiques, pédagogiques et techniques qui ont fondé sa démarche et guidé l'élaboration de son logiciel. Ce logiciel d'aide de révision des contenus se base sur un corpus de plus de 4000 unités lexicales et se divise en trois volets : 1. révision du vocabulaire ; 2 . révision du masculin/féminin/pluriel de certains substantifs et adjectifs ; 3 . révision des conjugaisons. L'enseignant a souligné que, outre la division en trois volets, le contenu de Revise o seu francês, était structuré en niveaux de difficulté (huit niveaux) de façon à veiller à une progression qui ne déroute pas l'apprenant. Ce logiciel qui vise l'autonomie de l'utilisateurapprenant est en cours d'évaluation auprès des étudiants afin que les observations recueillies puissent permettre d'identifier des améliorations.

L'intervention de Claudine Franchon (présentation du 10 octobre) intitulée Plurilinguisme et contacts de langues : stratégies d'intercompréhension en contexte virtuel MIRIADI a mis l'accent sur les interactions plurilingues en ligne intergroupes écrites et scénarisées dans le cadre du projet Miriadi (plateforme de formation à l'intercompréhension pour les langues romanes). L'auteure a choisi d'exploiter les interactions collectées sur les forums, issues d'une session de formation, en montrant comment les participants, regroupés en équipe locales puis en groupe de travail (GT) pratiquent l'intercompréhension pour construire ensemble/co-construire (perspective collaborative) un produit fini plurilingue et atteindre les objectifs d'apprentissage. L'auteure a pu illustrer de quelle 

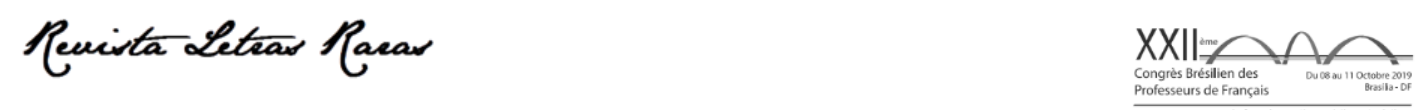

Actes du XXIlème Congrès brésilien des professeurs de français (dans Édition spéciale de la Revue Letras Raras 2020) ISSN : 2317-2347 - v. 9, Dossier Spécial (2020)

Todo o conteúdo da RLR está licenciado sob Creative Commons Atribuição 4.0 Internacional

façon les types de relations qui s'élaborent au travers des interactions bi-plurilingues ne se limitent pas à échanger des informations mais induisent la construction de formes interactives en fonction des interlocuteurs et des enjeux de l'échange. Est établie une forme de communication où chaque locuteur parle une langue différente (propre ou acquise) et comprend celle de l'autre, mais en mobilisant une série de stratégies communicatives et sociales pour faciliter la compréhension mutuelle et maintenir (grâce au scénario de la formation, influence des différentes phases de la formation et surtout des tâches à réaliser) un degré élevé de coopération entre les sujets.

\subsection{L'intercompréhension réceptive en contexte scolaire}

La communication de Josilene Pinheiro Mariz et Mariana de Normando intitulée L'intercompréhension et la littératie pour les enfants: Quand la théorie est mise en pratique (présentation du 9 octobre), soulève la question de l'importance de l'enseignement précoce des langues étrangères. En ce sens, les auteures ont présenté une recherche-action mettant en perspective les multiples bénéfices de l'intercompréhension des langues romanes associées à la problématique de la littératie dans le domaine éducationnel, pour un jeune public, dans le cadre d'une expérience conduite dans une école primaire du réseau public située à Alagoa Nova (État du Paraíba). Les auteures se sont interrogées sur la pertinence de l'intercompréhension des langues romanes comme facteur d'ouverture sur le monde et de plus-value pédagogique dans le processus de l'acquisition précoce du plurilinguisme en basant leur projet sur un travail littéraire plurilingue consistant en la compréhension de textes en français, en espagnol et en italien, et ce, dans une perspective de français langue étrangère (FLE). La conclusion de cette recherche-action a mis l'accent sur le bien-fondé de l'introduction de l'intercompréhension des langues romanes en contexte scolaire primaire où l'alternance des langues autour d'un groupe de langues de la même famille, prise en charge par les enseignants, est la clef d'une éducation pluriculturelle riche et novatrice dans laquelle l'exploitation du texte littéraire est centrale et démontre toute sa valeur éducative tout en soulevant de nombreux défis.

La communication de Karina Reyes (présentation du 9 octobre, a relaté une expérience de sensibilisation à l'intercompréhension des langues romanes se situant dans un Centre Interscolaire de Langues (CIL Gama) du District Fédéral. Intitulée Nouvelles approches pour l'enseignement de langues au DF : une expérimentation en intercompréhension à partir du livre "Les mots en jeu », l'auteure a exposé dans sa présentation comment l'intercompréhension 

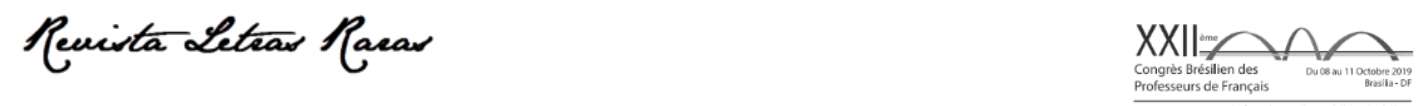

Actes du XXIlème Congrès brésilien des professeurs de français (dans Édition spéciale de la Revue Letras Raras 2020) ISSN : 2317-2347 - v. 9, Dossier Spécial (2020)

Todo o conteúdo da RLR está licenciado sob Creative Commons Atribuição 4.0 Internacional

pouvait s'insérer dans le cadre particulier du réseau public des CILs qui ont pour vocation, selon les directives de la politique linguistique mise en place par le Secrétariat d'État d'Éducation du District Fédéral (SEEDF), de promouvoir l'apprentissage des langues dans une perspective internationale de mobilité avec un focus sur le plurilinguisme et l'interculturalité. Sur la base de l'ouvrage cité qui se caractérise par un travail centré sur les stratégies lexicales de compréhension pour les langues romanes (français, espagnol, portugais), l'enseignante a mis en pratique une démarche plurilingue visant à développer chez l'apprenant lusophone la capacité de transfert et d'inférence au moyen de la manipulation de cartes de jeux trilingues (français, espagnol, portugais) selon l'approche des mots construits (morphologie dérivationnelle). L'auteure a insisté sur la dimension ludique de l'apprentissage, afin de produire un processus d'apprentissage actif versus passif qui a permis de mettre en œuvre une pédagogie de l'alternance des langues inscrite dans un rapport entre pensée, mot et action. C'est au travers d'une analyse qualitative que l'enseignante a exposé les bénéfices d'une telle approche contrastive auprès des apprenants de français langue étrangère (FLE) à partir d'une langue maternelle romane.

\subsection{L'intercompréhension réceptive, formation des adultes et professionnelle}

L'intervention de Mônica Duarte Serejo (présentation du 10 octobre), intitulée Palavras em jogo : Expérimentation d'accès au lexique plurilingue pour un public d'adultes, a également traité de la dimension lexicale de l'intercompréhension (IC). A partir de l'expérimentation de l'ouvrage Palavras em jogo - Intercompreensão : O vocabulario em uma perspectiva plurilingue, les travaux présentés ont mis en lumière comment à travers l'usage et la manipulation de mots selon une pédagogie de l'alternance des langues (français, espagnol, portugais), des apprenants adultes lusophones, dans un contexte d'apprentissage informel non institutionnalisé, ont mis en place des stratégies cognitives et élaboré des réflexions métalinguistiques contrastives permettant de développer les habilités de compréhension écrite pour une même famille de langues, les langues romanes. L'auteure a souligné, en présentant les témoignages des apprenants (selon une méthodologie qualitative), de quelle façon l'itinéraire pédagogique proposé avait pu avoir des répercussions sur l'apprentissage du français langue étrangère (FLE), en développant des compétences liées à la notion de transfert. 

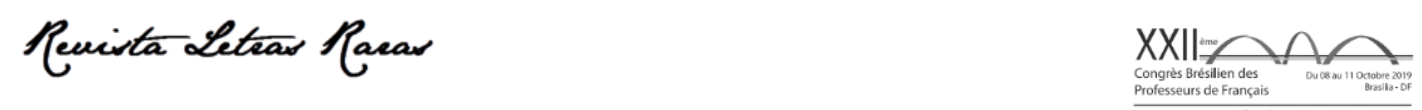

Actes du XXIlème Congrès brésilien des professeurs de français (dans Édition spéciale de la Revue Letras Raras 2020) ISSN : 2317-2347 - v. 9, Dossier Spécial (2020)

Todo o conteúdo da RLR está licenciado sob Creative Commons Atribuição 4.0 Internacional

\section{Références}

AUBERT, F.H. Introdução à metodologia da pesquisa terminológica bilingue. In: Cadernos de Terminologia, v.02, São Paulo : Humanitas Publicações, 1996

ARAÚJO E SÁ, M.H. ; DEGACHE, C. ; SPITA , D. Viagens em intercompreensão ... Quelques repères pour une « Galasaga ». In: ARAÚJO E SÁ, M. H. ; MELO-PFEIFER S. (Org.). Formação de formadores para a Intercompreensão. 1ed. Aveiro: Université d'Aveiro - CIDTFF-LALE, 2010, p.17-40.

BEBER, S. T. A língua portuguesa no computador. Campinas: Mercado das Letras, 2005

BLANCHET, P. Regard sociologique sur l'Intercompréhension entre langues différentes : enjeux théoriques et pistes sociodidactiques. In: GARBARINO, S.; DEGACHE C. (Org.). Intercompréhension en réseau. Scénarios, médiations, évaluations. 2ed. Lyon: Université Lumière Lyon2-CRTT, 2018, p.28-35.

CADDEO, S. ; JAMET, M.C. L'intercompréhension une autre approche pour l'intercompréhension des langues. Paris : Hachette Français langue étrangère, 2013 (collection « $F$ »).

CANDELIER, M. \& al. Cadre de référence pour les approches plurielles des langues et des cultures (CARAP), 1ed. Strasbourg/Graz : Conseil de l'Europe/ECML, 2007.

CONSEIL DE L'EUROPE. Un cadre européen commun de référence pour les langues : apprendre, enseigner, éduquer. 1ed. Strasbourg : Division des politiques linguistiques, 2001.

DEGACHE, C. Nouvelles perspectives pour l'intercompréhension (Afrique de l'Ouest et Caraïbe) et évolution du concept. In: ARAÚJO E SÁ, M. H.; HIDALGO DOWNING, S.; MELO-PFEIFER S.; SÉRÉ, A.; VELA DELFA C. (Org.). Intercompreensão em línguas românicas: conceitos, práticas, formação. 1ed. Aveiro: Université d'Aveiro - CIDTFF, 2009, p.82-94.

DEGACHE, C.; GARBARINO, S. Itinéraires pédagogiques de l'alternance des languesl'intercompréhension. 1ed. Grenoble : UGA, 2017 (collection didaskein).

DEVELOTTE, C. ; KERN R. ; LAMY, M.N. Décrire la conversation en ligne. Lyon : ENS Éditions, 2011.

ESCUDÉ, P. ; JANIN, P. Le point sur l'intercompréhension, clé du plurilinguisme. 1ed. Paris : CLE International, 2010.

ESCUDÉ, P. L'intercompréhension et la triple répartition. Redinter-intercompreensão, n.3, p. 113130, 2011.

FEUILLET, J. Les enjeux d'une sensibilisation très précoce aux langues étrangères en milieu institutionnel. Paris : CRINI, 2008.

FRANCHON, C. ; STRHLER, R. Palavras em jogo - Intercompreensão: O vocabulario em uma perspectiva plurilingue. Campinas : Pontes, 2017.

GOANAC'H, D. L'Apprentissage précoce d'une langue étrangère: Le point de vue de la psycholinguistique. Paris : Hachette Education, 2015 (collection « Profession Enseignant »).

LEGROS, D. ; CRINON, J. Psychologies des apprentissages et multimédia. Paris: Armand-Colin, 2002. 

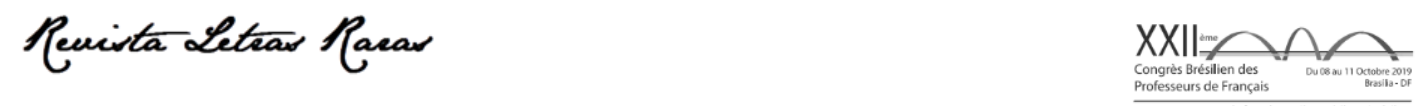

Actes du XXIlème Congrès brésilien des professeurs de français (dans Édition spéciale de la Revue Letras Raras 2020)

ISSN : 2317-2347 - v. 9, Dossier Spécial (2020)

Todo o conteúdo da RLR está licenciado sob Creative Commons Atribuição 4.0 Internacional

MOORE, D.; GAJO, L. Introduction to French voices on plurilingualism and pluriculturalism: Theory, significance and perspectives, International Journal of Multilingualism, v.6, n.2, p.137-153, 2009.

LEWIS, M.P. ; SIMONS, G. F.; FENNING, C.D. Ethnologue : Languages of the World,. 1ed. Dallas: SIL International, 2015.

MATTHEY, M. ; DE PIETRO, J. F. La société plurilingue: utopie souhaitable ou domination acceptée ? In: Plurilinguisme : " contact » ou « conflit » de langues? Paris : L'Harmattan, 1997, p. 133-190.

MEISSNER, F. J. \& al. Les sept tamis: lire les langues romanes dès le départ. Avec une introduction à la didactique de l'eurocompréhension. 1ed. Aachen: Shaker-Verlag, 2004, (collection Ediciones EuroCom).

MOLINIÉ, M. ; MOORE, D. Les littératies : une notion en Questions en didactique des langues (NeQ). Recherches en didactique des langues et des cultures, Cergy-Pontoise, v.9, n.2, 2012.

NISSEN, E. ; POYET, F. ; SOUBRIÉ, T. Interagir en ligne. Grenoble : ELLUG, 2011.

PORQUIER, R. Communication exolingue et apprentissage des langues. In: Acquisition d'une langue étrangère III. 1ed. Paris-Neuchâtel: Presses Universitaires de Vincennes - Centre de linguistique appliquée, 1984.

PY, B. Les stratégies d'interaction en situation d'interaction. In: GAONAC'H, D. (Org.). Acquisition et utilisation d'une langue étrangère : l'approche cognitive, Le français dans le monde, recherches et applications. Paris : Hachette (collection « F »), 1990, p.81-88.

SILVA, (DA) R. Intercompreensão e interculturalidade entre discursos competentes e outras falas. In: GARBARINO, S. ; DEGACHE C. (Org.). Intercompréhension en réseau. Scénarios, médiations, évaluations. 2ed. Lyon: Université Lumière Lyon2-CRTT, 2018, p.141-150. 